\title{
Widespread Metastasis
}

National Cancer Institute

\section{Source}

National Cancer Institute. Widespread Metastasis. NCI Thesaurus. Code C162651.

The spread of a malignant tumor in multiple distant anatomic sites. 